\title{
Characteristics of Confirmed Novel Coronavirus Disease Cases at Shanghai East Hospital: A Retrospective Single-Center Study
}

\section{Chenghui Fan}

shanghai east hospital

Lihong Qu

shanghai east hospital

Zhongliang Guo

Shanghai East Hospital

Jianhua Zhang

shanghai east hospital

Jun Wang

shanghai east hospital

Qin Li

Shanghai East Hospital

Sheng Kang

Shanghai East Hospital

Yue Wang

Shanghai East Hospital

Lei $\mathrm{Xu}$

Shanghai East Hospital

Wei Wang

Shanghai East Hospital

Wenzhong Yang

Shanghai East Hospital

Ying Zhou

Shanghai East Hospital

Yuanyuan Wang

Shanghai East Hospital

Ying Hao

Shanghai East Hospital

Ruilin Li

Shanghai East Hospital

Feng Sun 
Shanghai East Hospital

Junli Xue ( $D$ 1310666xuejunli@tongji.edu.cn )

Shanghai East Hospital https://orcid.org/0000-0002-0553-6993

\section{Research}

Keywords: COVID-19, fever outpatient clinic, isolation, pandemic, quarantine

Posted Date: June 19th, 2020

DOI: https://doi.org/10.21203/rs.3.rs-33689/v1

License: (c) (1) This work is licensed under a Creative Commons Attribution 4.0 International License. Read Full License 


\section{Abstract}

Background: Since the outbreak of the novel coronavirus disease (COVID-19), the fever outpatient clinic has been open in Shanghai East Hospital (SEH). We analyzed the data for all 4,699 patients from SEH and the 27 confirmed COVID-19 cases among them to determine the clinical and epidemiological characteristics of confirmed COVID-19 cases identified in the SEH.

Methods: Data were collected for patients who visited the fever outpatient clinic in the SEH between January 23 and April 30, 2020. We compared the characteristics of confirmed cases, including age, occupation, area, symptoms, laboratory results, and computed tomography (CT) scans, by month.

Results: By April 30, 4,699 patients had visited the fever outpatient clinic of the SEH; of those, 27 (0.57\%) were confirmed COVID-19 cases. Among the confirmed domestic cases identified between January and February, four of five were from Wuhan, Hubei. Following the spread of the epidemic to other parts of the world, all confirmed cases identified in March-April were cases of individuals who were returning from abroad, mainly Chinese students living abroad. Further, all cases were from outside Shanghai, and no local residents were diagnosed in the clinic. Symptoms, laboratory tests, and CT scans were consistent with previous literature reports of positive COVID-19 cases.

Conclusions: Given the necessity to control the spread of this epidemic domestically and abroad, the focus of COVID-19 prevention and control has shifted. In Shanghai, measures taken to prevent COVID-19 spread were very successful. Early isolation and quarantine are necessary and effective measures.

\section{Background}

Since the outbreak of a cluster of pneumonia cases of unknown origin in December 2019, this disease has spread rapidly across the world [1]. The pathogen has been identified by reverse transcriptionpolymerase chain reaction (RT-PCR) as a novel enveloped RNA beta-coronavirus from samples of patients' lower respiratory tracts [1-2]. On February 11, 2020, the World Health Organization (WHO) named the new coronavirus as severe acute respiratory syndrome coronavirus 2 (SARS-CoV-2) and the disease caused by the virus as coronavirus disease (COVID-19) [3]. Due to its high infectivity, COVID-19 spread quickly in China and worldwide. Consequently, on March 11, 2020, the WHO declared a pandemic [4]. As of May 9, 2020, the cumulative number of COVID-19 infections worldwide has exceeded 3.8 million [5]. In China, COVID-19 was brought under control within 2 months with effective preventive measures.

As China's largest city that bridges domestic and overseas affairs, Shanghai faced both domestic and global epidemic pressure. Since the COVID-19 pandemic, Shanghai has mainly experienced imported cases, having the second-largest number of imported cases in the mainland of China. However, Shanghai adopted strict measures to prevent and control the spread of COVID-19 to reduce its transmission.

Shanghai East Hospital (SEH) is one of the largest general tertiary care hospitals in Pudong New District, playing an important role during the epidemic prevention and control in Shanghai. On January 23, 2020 
when Wuhan was locked down, SEH, in response to the national and government policies, opened the fever outpatient clinic which undertook the screening, triage, and treatment of COVID-19 patients. Furthermore, as SEH is located close to the Pudong International Airport, the hospital committed to the responsibility of screening and treating imported cases. Herein, we analyze the data for all patients and COVID-19 confirmed cases identified between January 23 and April 30, 2020 in the fever outpatient clinic of the SEH in order to elucidate the epidemic situation in the SEH and Shanghai.

\section{Methods}

\section{Study design}

This study is a retrospective single-center study that analyzes the data collected for 4,699 patients who visited the fever outpatient clinic on the SEH between January 23 and April 30, 2020. All patients with a sublingual temperature higher than $37.3^{\circ} \mathrm{C}$ as assessed in the general outpatient department were referred to the fever outpatient clinic for COVID-19 screening. Patients who had a sublingual temperature higher than $37.3^{\circ} \mathrm{C}$ and were identified at either the Pudong International Airport or a quarantine point, or those who had a cough, headache, sore throat, or other symptoms of infection were also sent to the fever clinic by ambulance. COVID-19 was diagnosed according to the guidelines for the diagnosis and treatment program published by the National Health Commission of China (5th -7th edition, in Chinese) [6-8]. This study was approved by the ethics committee of the SEH. Given the retrospective design, the need for written informed consent was waived.

\section{Procedures and data collection}

For all patients, epidemiological and demographic data as well as symptoms and signs of disease were collected, and routine blood examination, inflammation blood tests, lung CT scans, and nucleic acid screening for COVID-19 were performed routinely at the SEH. All data were obtained from patient medical records. Fever was defined as a sublingual temperature of $37.3^{\circ} \mathrm{C}$ or higher. Lymphocytopenia was defined as a lymphocyte count of less than $1.1 \times 10^{9} / \mathrm{L}$. Based on the results of the laboratory tests and lung CT scans, suspected cases underwent consultation with a specialist. If COVID-19 could not be excluded, the case was referred to the Chinese center for Disease prevention and Control (CDC) for further nucleic acid testing. Samples of post-nasal swabs were collected for the nucleic acid test, and COVID-19 was confirmed by RT-PCR. Cases with once positive RT-PCR result was defined as confirmed cases. The confirmed cases were transferred to the Shanghai Public Health Clinical Center by special ambulances for further treatment.

\section{Statistical analysis}

The variables that were analyzed included demographic and epidemiological data for all patients in the fever outpatient clinic as well as the main symptoms, signs, laboratory examination results, and imaging examination results of the confirmed COVID-19 patients. Categorical and continuous data were analyzed using descriptive statistics. Categorical variables are presented as frequency rates and percentages, while 
continuous variables are expressed as means \pm standard deviations. Means for continuous variables that were normally distributed were compared using one-way ANOVA. Categorical variables were analyzed using the Wilcoxon rank-sum and Kruskal-Wallis tests. All statistical analyses were performed using SPSS 23.0, and $p<0.05$ was considered statistically significant.

\section{Results}

\section{Positive rates and demographic data}

Between January 23 and April 30, 2020, a total of 4,699 patients visited the fever outpatient clinic on the SEH; of those patients, 27 were diagnosed as confirmed COVID-19 cases $(0.57 \%)$. The number of patients who visited the hospital between January 23 and February 29, 2020 was 2,344, and 5 positive cases were identified $(0.21 \%)$. In March, 1,379 patients visited the fever outpatient clinic and 15 were diagnosed as positive for COVID-19 (1.09\%). Finally, in April, 976 patients visited the fever clinic and 7 tested positive for COVID-19 (0.72\%). Among the 27 confirmed cases, 12 were men (44.44\%) and 15 were women (55.56\%).

Age and occupation of the patients varied by month. The average age of the 27 confirmed patients was $32.85 \pm 17.43$, while the average age of the 5 confirmed patients in January and February was $51.00 \pm$ 17.78. The mean ages of the confirmed cases from March and April were 29.13 \pm 15.11 and $27.86 \pm$ 15.34 , respectively. As for the occupations of the patients, the majority of the patients in January and February were retirees (2 [40.00\%]) and employees (2 [40.00\%]). Among the cases identified in March and April, the majority were students (8 [53.33\%] and 5 [71.43\%], respectively). Age and occupation differed significantly by month $(p<0.05)$. Such differences may be due to the various regions that the patients came from at different times of the epidemic (Table 1 and Fig. 1). 
Table 1

\begin{tabular}{|c|c|c|c|c|c|}
\hline Characteristics & $\begin{array}{l}\text { January \& } \\
\text { February }\end{array}$ & March & April & All cases & $\begin{array}{l}p- \\
\text { value }\end{array}$ \\
\hline Total number & 2344 & 1379 & 976 & 4699 & \\
\hline $\begin{array}{l}\text { Confirmed cases } \\
\text { (rate\%) }\end{array}$ & $5(0.21)$ & 15 (1.09) & $7(0.72)$ & $27(0.57)$ & \\
\hline Sex, n (\%) & & & & & 0.964 \\
\hline Male & $2(40.00)$ & $7(46.67)$ & $3(42.86)$ & $12(44.44)$ & \\
\hline Female & $3(60.00)$ & $8(53.33)$ & $4(57.14)$ & $15(55.56)$ & \\
\hline Age, n (\%) & $51.00 \pm 17.78$ & $\begin{array}{l}29.13 \pm \\
15.11\end{array}$ & $\begin{array}{l}27.86 \pm \\
15.34\end{array}$ & $\begin{array}{l}32.85 \pm \\
17.43\end{array}$ & 0.028 \\
\hline$\nabla 12 Y$ & $0(0.00)$ & $1(6.67)$ & $0(0.00)$ & $1(3.70)$ & \\
\hline $12-44 Y$ & $2(40.00)$ & $11(73.33)$ & $6(85.71)$ & 19 (70.37) & \\
\hline $45-64 Y$ & $2(40.00)$ & $3(20.00)$ & $1(14.29)$ & $6(22.22)$ & \\
\hline$\geq 65 Y$ & $1(20.00)$ & $0(0.00)$ & $0(0.00)$ & $1(3.70)$ & \\
\hline Occupation, n (\%) & & & & & 0.002 \\
\hline Retiree & $2(40.00)$ & $1(6.67)$ & $0(0.00)$ & $3(11.11)$ & \\
\hline Employee & $2(40.00)$ & $2(13.33)$ & $0(0.00)$ & $4(14.81)$ & \\
\hline Service & $1(20.00)$ & $3(20.00)$ & $0(0.00)$ & $4(14.81)$ & \\
\hline Student & $0(0.00)$ & $8(53.33)$ & $5(71.43)$ & $13(48.15)$ & \\
\hline Unemployed & $0(0.00)$ & $1(6.67)$ & $2(28.57)$ & $3(11.11)$ & \\
\hline Region, n (\%) & & & & & $\bowtie 0.001$ \\
\hline Shanghai residents & $0(0.00)$ & $0(0.00)$ & $0(0.00)$ & $0(0.00)$ & \\
\hline Non-resident & $5(100.00)$ & $0(0.00)$ & $0(0.00)$ & $5(18.52)$ & \\
\hline Abroad & $0(0.00)$ & $15(100.00)$ & $7(100.00)$ & $22(81.48)$ & \\
\hline Nationality, n (\%) & & & & & 0.435 \\
\hline Chinese & $5(100.00)$ & $13(86.67)$ & $7(100.00)$ & 25 (92.59) & \\
\hline Foreigner & $0(0.00)$ & $2(13.33)$ & $0(0.00)$ & $2(7.41)$ & \\
\hline \multicolumn{6}{|l|}{ Symptoms, n (\%) } \\
\hline Temperature & $37.50 \pm 1.01$ & $37.14 \pm 0.88$ & $37.33 \pm 0.43$ & $37.26 \pm 0.80$ & 0.675 \\
\hline
\end{tabular}




\begin{tabular}{|llllll|}
\hline Characteristics & $\begin{array}{l}\text { January \& } \\
\text { February }\end{array}$ & March & April & All cases & $\begin{array}{c}\text { p- } \\
\text { value }\end{array}$ \\
\hline $37.3-38.0^{\circ} \mathrm{C}$ & $1(20.00)$ & $4(26.67)$ & $3(42.86)$ & $8(29.63)$ & \\
\hline $38.1-39.0^{\circ} \mathrm{C}$ & $1(20.00)$ & $3(20.00)$ & $0(0.00)$ & $4(14.81)$ & \\
\hline$\otimes 39.0^{\circ} \mathrm{C}$ & $0(0.00)$ & $0(0.00)$ & $0(0.00)$ & $0(0.00)$ & \\
\hline Cough & $1(20.00)$ & $6(40.00)$ & $0(0.00)$ & $7(25.93)$ & 0.140 \\
\hline Pharyngalgia & $0(0.00)$ & $2(13.33)$ & $1(14.29)$ & $3(11.11)$ & 0.273 \\
\hline Headache & $1(20.00)$ & $2(13.33)$ & $0(0.00)$ & $3(11.11)$ & 0.522 \\
\hline Muscle ache & $0(0.00)$ & $1(6.67)$ & $0(0.00)$ & $1(3.70)$ & 0.670 \\
\hline Rhinorrhea & $1(20.00)$ & $0(0.00)$ & $0(0.00)$ & $1(3.70)$ & 0.111 \\
\hline Laboratory test & & & & & \\
\hline White blood cells & $6.17 \pm 1.99$ & $6.25 \pm 1.94$ & $7.29 \pm 1.35$ & $6.51 \pm 1.81$ & 0.431 \\
\hline Normal & $5(100.00)$ & $15(100.00)$ & $7(100.00)$ & $27(100.00)$ & \\
\hline Abnormal & $0(0.00)$ & $0(0.00)$ & $0(0.00)$ & $0(0.00)$ & \\
\hline Lymphocytes & $1.58 \pm 0.41$ & $2.00 \pm 1.43$ & $1.84 \pm 0.62$ & $1.89 \pm 1.11$ & 0.770 \\
\hline Normal & $5(100.00)$ & $11(73.33)$ & $5(71.43)$ & $21(77.78)$ & \\
\hline Lymphopenia & $0(0.00)$ & $4(26.67)$ & $2(28.57)$ & $6(22.22)$ & \\
\hline Lung CT, $n$ (\%) & $1(20.00)$ & $5(33.33)$ & $5(71.43)$ & $11(40.74)$ & \\
\hline Normal & $4(80.00)$ & $10(66.67)$ & $2(28.57)$ & $16(59.26)$ & \\
\hline Abnormal & & & & \\
\hline CT, computed tomography) & Y, years & & & & \\
\hline
\end{tabular}

\section{Regional distribution of COVID-19 confirmed cases from the SEH fever outpatient clinic}

In terms of regional distribution, none of the 27 confirmed patients were residents of Shanghai. COVID-19 positive cases came from a variety of regions at different epidemic times. All five confirmed cases that were identified between January and February were infected domestically; while four cases were residents of Wuhan (80.00\%), one was of Hunan province (20.00\%). All cases identified in March and April were from abroad. Among the 15 cases that were identified in March, 13 were Chinese residents who returned to Shanghai from abroad (86.67\%) and 2 were foreigners $(13.33 \%)$. All seven cases detected in April were Chinese residents who returned to Shanghai from abroad. 
Cases identified in March were mainly from Europe, including six cases from the United Kingdom $(40.00 \%)$, two cases from France (13.33\%), and one case each from Italy, Switzerland, and Russia $(6.67 \%)$. The other four patients had returned from the United States $(26.67 \%)$. All seven cases identified in April returned from abroad: five from the United Kingdom (71.43\%), one from Brazil, and one from Russia (14.29\%) (Fig. 2). The majority of the cases that returned from abroad came from the United Kingdom and were predominantly Chinese students who were studying abroad (Fig. 3).

\section{Symptoms and laboratory tests analysis}

The main symptom of the 27 confirmed patients was fever (12 [44.44\%]), with an average body temperature of $37.26 \pm 0.80^{\circ} \mathrm{C}$. Other common symptoms included cough (7 [25.93\%]), pharyngalgia (3 [11.11\%]), headache (3 [11.11\%]), muscle ache and rhinorrhea (1 [3.70\%]) (Fig. 4).

The laboratory tests revealed that the white blood cell counts of all 27 COVID-19 confirmed patients were normal $\left(6.51 \pm 1.81 \times 10^{9} / \mathrm{L}\right)$ and there were no significant differences among different months. Lymphopenia was observed in six patients $(22.22 \%)$, and the absolute number of lymphocytes was normal in the remaining 21 patients, with an average lymphocyte count of $1.89 \pm 1.11 \times 10^{9} / \mathrm{L}$. There were 16 (59.26\%) patients with abnormal lung CT scans (Fig. 5). The lung CT findings of most patients were marked by multiple mottling and ground-glass opacities in the bilateral lung lobe. Typical lung CT findings are shown in Fig. 6. According to the guidelines of the diagnosis and treatment program issued by the National Health Commission of China, all 27 confirmed patients had either mild illness or normal pneumonia. There were no cases of severe pneumonia or acute respiratory distress syndrome.

\section{Discussion}

Since its outbreak in December 2019, COVID-19 has affected nearly 3.8 million people in more than 200 countries and regions [5]. With the spread of the epidemic, all countries are currently experiencing pressure to control and prevent the further spread of COVID-19. As an international hub in China, Shanghai faces severe pressure to control and prevent further spread of the epidemic due to its large population flow.

The analyses presented herein demonstrate that the focus to control and prevent the spread of COVID-19 in Shanghai has gradually shifted from domestic to imported cases; thus, the characteristics of confirmed cases have gradually changed. In January and February, the COVID-19 epidemic was mainly contained within the Chinese mainland. Patients with fever who visited the SEH between January and February were mainly domestic patients, and four of the five confirmed cases were from Wuhan. In March 2020 , with the lockdown of Wuhan and the control of the epidemic in China, the confirmed cases decreased significantly in mainland China. However, by then, the epidemic had spread to Europe and other parts of the world. A large number of Chinese citizens living abroad flocked home, exerting new pressure on China. This group of citizens consisted mainly of students who were studying abroad. Our data indicate that among the cases identified in March and April, 22 cases returned from abroad: 11 were 
from the United Kingdom and 10 of them were Chinese citizens living in the United Kingdom as overseas students.

As one of China's largest cities, Shanghai had the second-highest number of imported cases in China as of May 9, 2020. However, despite the enormous pressure both domestically and globally, Shanghai has taken positive and effective measures to prevent and control the spread of COVID-19. Among all patients from the fever outpatient clinic that visited the SEH, none of the confirmed cases were local residents. Additionally, Shanghai did not report any infections among its medical staff [9]. Currently, the Pudong customs classifies people returning to Shanghai, from abroad, according to their point of origin. People returning from high-risk countries and regions are quarantined for 14 days. During quarantine, body temperature is monitored daily. If symptomatic people are observed to have a fever or cough, they are sent to nearby fever outpatient clinics for COVID-19 screening in a timely manner [10], and confirmed cases are transferred to the Shanghai Public Health Clinical Center for further treatment. Regarding the prevention and control of COVID-19, we have implemented strict measures to identify the source of infection among people entering Shanghai, cut off the route of transmission, and protect susceptible populations.

The symptoms of the 27 confirmed patients reported in this study are consistent with the symptoms reported in the extant literature $[11,12]$. The main symptoms included fever, cough, pharyngalgia, and headache. Further, laboratory tests revealed normal white blood cell counts and lymphopenia. Lung CT scans indicated that more than half of the patients had remarkable lung CT imaging findings. According to the new coronavirus pneumonia diagnosis and treatment program implemented by the National Health Commission, the lung CT scans of COVID-19-positive patients indicate ground-glass opacity findings, which are typical changes observed in viral pneumonia [13]. The diagnosis mainly relies on the etiology of the disease and the COVID-19 RT-PCR test; patients who test positive are considered confirmed cases.

Specific treatment and prevention options, such as targeted antiviral drugs, nucleoside analogs, chloroquine, protease inhibitors, and vaccines, were not available for COVID-19 due to the lack of clinical evidence during the timeframe for which the study was conducted [14]. Instead, clinicians in China focused on traditional public health outbreak response tactics, such as isolation, quarantine, social distancing, and community containment. Patients with mild illness and normal pneumonia were mainly treated with supportive care. On May 1, 2020, the Food and Drug Administration (FDA) granted emergency access to Remdesivir, a nucleotide analog prodrug that inhibits viral RNA polymerases, for patients with severe COVID-19 and children; however, controversy regarding Remdesivir treatment for COVID-19 remains. The phase III clinical study conducted in China indicated that Remdesivir did not significantly improve the time to clinical improvement, mortality, or time to clearance of virus in patients with serious COVID-19 compared with placebo. However, among patients receiving Remdesivir or placebo within 10 days of symptom onset in the intention to treat (ITT) population, those receiving Remdesivir had a numerically faster time of clinical improvement than those receiving placebo (median 18.0 days vs. 23.0 days, hazard ratio (HR) 1.52) [15]. In a study of Remdesivir compassionate use for severe COVID-19 
cases, clinical improvement was observed in 36 of 53 patients (68\%). Nevertheless, to determine the efficacy of Remdesivir, further randomized, placebo-controlled trials are needed [16].

A randomized, double-blind, placebo-controlled study of 1,063 COVID-19 patients that was initiated by the National Institute of Allergy and Infectious Diseases (NIAID) determined that patients treated with Remdesivir had a $31 \%$ faster time to recovery than those who received a placebo ( 11 days vs 15 days, $p<$ 0.001). Results also suggest a survival benefit, with a mortality rate of $8.0 \%$ for the group receiving Remdesivir versus $11.6 \%$ for the placebo group $(p=0.059)$ [17]. Furthermore, there is no evidence for the role of traditional Chinese medicine and other antiviral drugs in the treatment of COVID-19.

Corticosteroids for COVID-19 treatment are neither recommended by the WHO nor by the China National Health Commission $[8,18]$.

This study, however, has several limitations. First, we did not obtain more detailed patient information, particularly regarding clinical outcomes, because it was unavailable at the time of analysis. Second, a larger sample size is required to be more representative of the population of Shanghai. Third, the confirmed cases were transferred to the Shanghai Public Health Clinical Center, and limited treatment data were collected there.

\section{Conclusions}

The results presented herein suggest that, since the beginning of the COVID-19 outbreak, the target population for the prevention and control of the epidemic in Shanghai has shifted as COVID-19 continues to spread across the world. However, overall, the prevention and control measures adopted in Shanghai were positive and effective. Fever outpatient clinics in Pudong New District have played a pivotal role in the screening, triage, and treatment of confirmed cases and have contributed to the prevention and control of COVID-19. The clinical manifestations of the confirmed patients reported herein were consistent with the Chinese guidelines and relevant literature reports. However, in terms of treatment, there is no clear and effective drug to eliminate the virus. Early diagnosis and isolation are rudimentary yet effective strategies to mitigate the transmission of the virus.

\section{Abbreviations}

ANOVA: Analysis of variance

CDC: Center for Disease prevention and Control

COVID-19: coronavirus disease 2019

CT: computed tomography

FDA: Food and Drug Administration

HR: Hazard Ratio

Page 10/17 
NIAID: National Institute of Allergy and Infectious Diseases

RT-PCR: reverse transcription-polymerase chain reaction

SEH: Shanghai East Hospital

WHO: World Health Organization

\section{Declarations}

\section{Ethics approval and consent to participate}

The study was approved by the ethics committee of the SEH. As this study has a retrospective design, written informed consent was waived.

\section{Consent for publication}

Not applicable.

\section{Availability of data and materials}

The datasets used and analyzed during the current study are available from the corresponding author on reasonable request.

\section{Competing interests}

The authors declare that they have no competing interests.

\section{Funding}

This study was funded by the Outstanding Clinical Discipline Project of Shanghai Pudong (No. PWYgy2018-02). Science and Technology Commission of Shanghai Municipality (Grant number: 17411968800 and 19DZ1910502, to JL Xue).

\section{Authors' contributions}

$\mathrm{FCH}, \mathrm{QLH}$, and SF collected the epidemiological and clinical data and processed statistical data. GZL and ZJH determined the diagnoses of confirmed cases. LQ, KS, WY, XL, WW, YWZ, ZY, WJ, WYY, HY, and LRL screened all the positive cases. FCH and XJL drafted the manuscript. XJL and SF revised the final manuscript. XJL is responsible for summarizing all data related to the virus. QLH is responsible for 
summarizing all epidemiological and clinical data. All the authors were physicians of Shanghai East Hospital that worked in the fever outpatient clinic voluntarily during the epidemic.

\section{Acknowledgments}

We thank all patients involved in the study. We would like to thank Editage (www.editage.cn) for English language editing.

\section{References}

1. Zhu N, Zhang D, Wang W, Li X, Yang B, Song J, et al. A novel coronavirus from patients with pneumonia in China, 2019. N Engl J Med. 2020;382:727-33.

2. Wu F, Zhao S, Yu B, Chen Y M, Wang W, Song Z G, et al. A new coronavirus associated with human respiratory disease in China. Nature. 2020;579:265-9.

3. World Health Organization. Coronavirus disease (COVID-19) outbreak. World Health Organization. 2020. https://www.who.int/emergencies/diseases/novel-coronavirus-2019. Accessed 09 May 2020

4. World Health Organization. Coronavirus disease (COVID-19) pandemic. World Health Organization. 2020. https://www.who.int/emergencies/diseases/novel-coronavirus-2019. Accessed 09 May 2020

5. World Health Organization. Coronavirus disease 2019 (COVID-19) situation report-110. World Health Organization. 2020. https://www.who.int/docs/default-source/coronaviruse/situationreports/20200509covid-19-sitrep-110.pdf?sfvrsn=3b92992c_4. Accessed 09 May 2020

6. National Health Commission of China. New coronavirus pneumonia diagnosis and treatment program (7th ed.) (in Chinese). 2020.

http://www.nhc.gov.cn/yzygj/s7652m/202003/a31191442e29474b98bfed5579d5af95.shtml. Accessed 09 May 2020

7. National Health Commission of China. New coronavirus pneumonia diagnosis and treatment program (5th ed.) (in Chinese). 2020.

http://www.nhc.gov.cn/yzygj/s7652m/202002/e84bd30142ab4d8982326326e4db22ea.shtml. Accessed 09 May 2020

8. National Health Commission of China. New coronavirus pneumonia diagnosis and treatment program (6th ed.) (in Chinese). 2020. http://www.nhc.gov.cn/yzygj/s7652m/202002/54e1ad5c2aac45c19eb541799bf637e9.shtml. Accessed 09 May 2020

9. Chen J, Qi T, Liu L, Ling Y, Qian Z, Li T, et al. Clinical progression of patients with COVID-19 in Shanghai, China. J Infect. 2020;80:e1-6.

10. Pudong International Airport screening (in Chinese). 2020. http://finance.sina.com.cn/roll/2020-0411/doc-iirczymi5626797.shtml. Accessed 09 May 2020 
11. Rothan HA, Siddappa N. The epidemiology and pathogenesis of coronavirus disease (COVID-19) outbreak. J Autoimmun. 2020;109:102433.

12. Lake M A. What we know so far: COVID-19 current clinical knowledge and research. Clin Med (Lond). 2020;20:124-7.

13. Chen N, Zhou M, Dong X, Qu J, Gong F, Han Y, et al. Epidemiological and clinical characteristics of 99 cases of 2019 novel coronavirus pneumonia in Wuhan, China: a descriptive study. Lancet. 2020;395:507-13.

14. Ahn D G, Shin H J, Kim M H, Lee S, Kim H S, Myoung J, et al. Current status of epidemiology, diagnosis, therapeutics, and vaccines for novel coronavirus disease 2019 (COVID-19). J Microbiol Biotechnol. 2020;30:313-24.

15. Wang Y, Zhang D, Du G, Du R, Zhao J, Jin Y, et al. Remdesivir in adults with severe COVID-19: a randomized, double-blind, placebo-controlled, multicentre trial. Lancet. 2020;395:1521-86.

16. Grein J, Ohmagari N, Shin D, Diaz G, Asperges E, Castagna A, et al. Compassionate use of remdesivir for patients with severe Covid-19. N Engl J Med. 2020:1-10.

17. John HB, Kay MT, Lori ED, Aneesh KM, Barry SZ, Andre CK, et al. Remdesivir for the treatment of Covid-19 - Preliminary Report. N Engl J Med. 2020. doi:10.1056/NEJMoa2007764.

18. World Health Organization. Clinical management of severe acute respiratory infection when novel coronavirus (nCoV) infection is suspected. Interim Guidance. World Health Organization. 2020. https://www.who.int/docs/default-source/coronaviruse/clinical-management-of-novel-cov.pdf. Accessed 09 May 2020

\section{Figures}



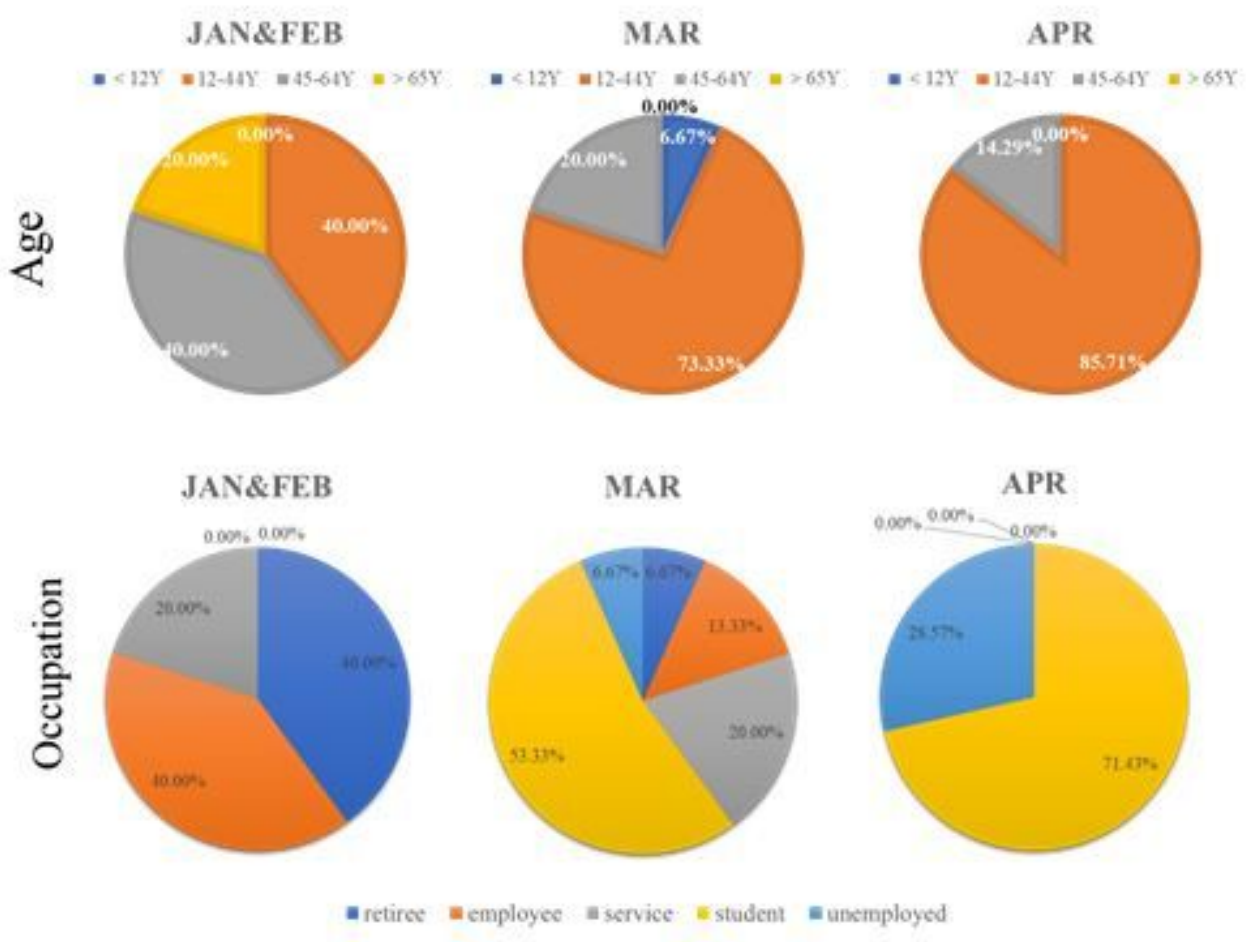

\section{Figure 1}

Age and occupation analysis of all COVID-19 confirmed cases from the SEH fever outpatient clinic. Due to the fact that the patients came from various regions at different times of the epidemic, age and occupation of the patients varied by month. The mean ages of the confirmed cases from March and April were much younger than those in January and February. Students were major occupation of the patients identified in March and April, different from those in January and February who were mostly retirees and employees. COVID-19, coronavirus disease 2019; SEH, Shanghai east hospital 


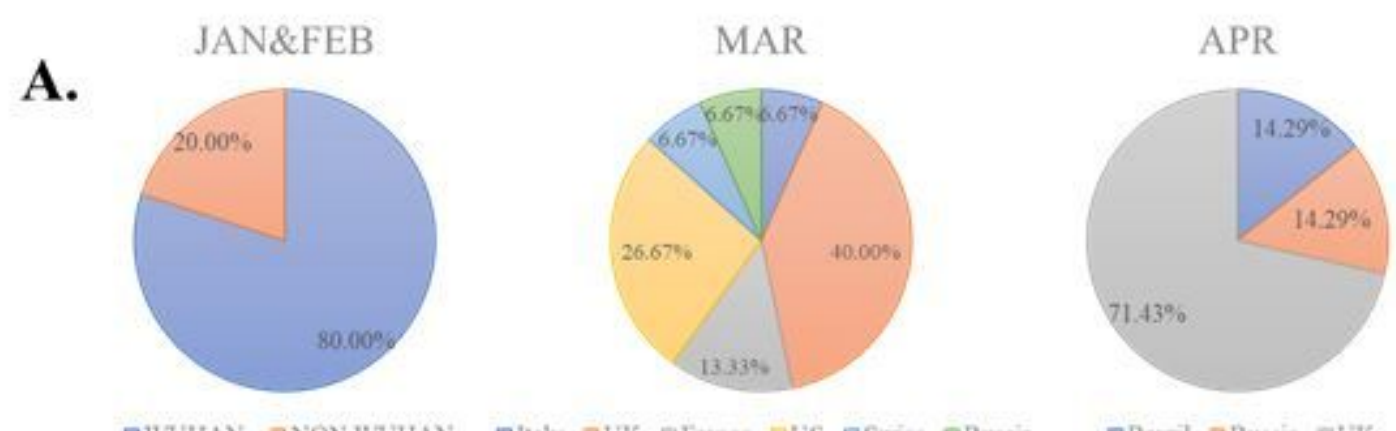

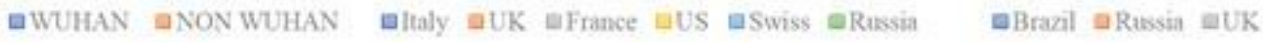

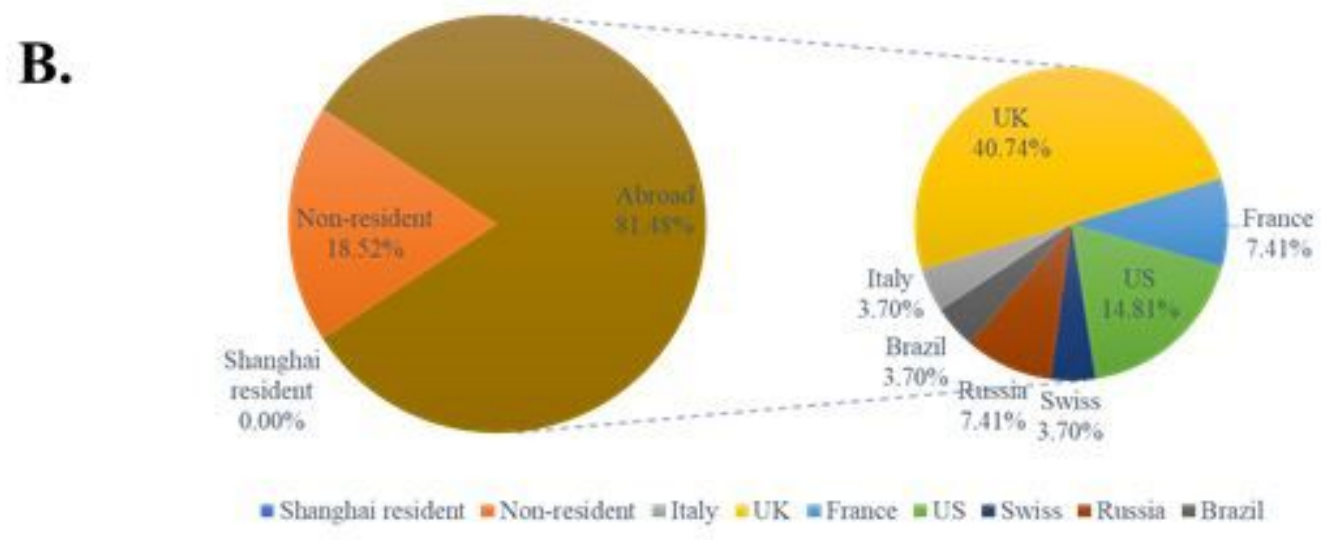

\section{Figure 2}

Regional distribution of confirmed case from SEH. A. COVID-19 positive cases came from various regions at different times. In January and February, all cases were infected domestically. All cases identified in March and April were from abroad, who were mainly Chinese residents returned to Shanghai. B. Among all 27 confirmed case, 22[81.48\%] were from abroad. The majority of imported cases returned from the United Kingdom. COVID-19, coronavirus disease 2019; SEH, Shanghai east hospital 


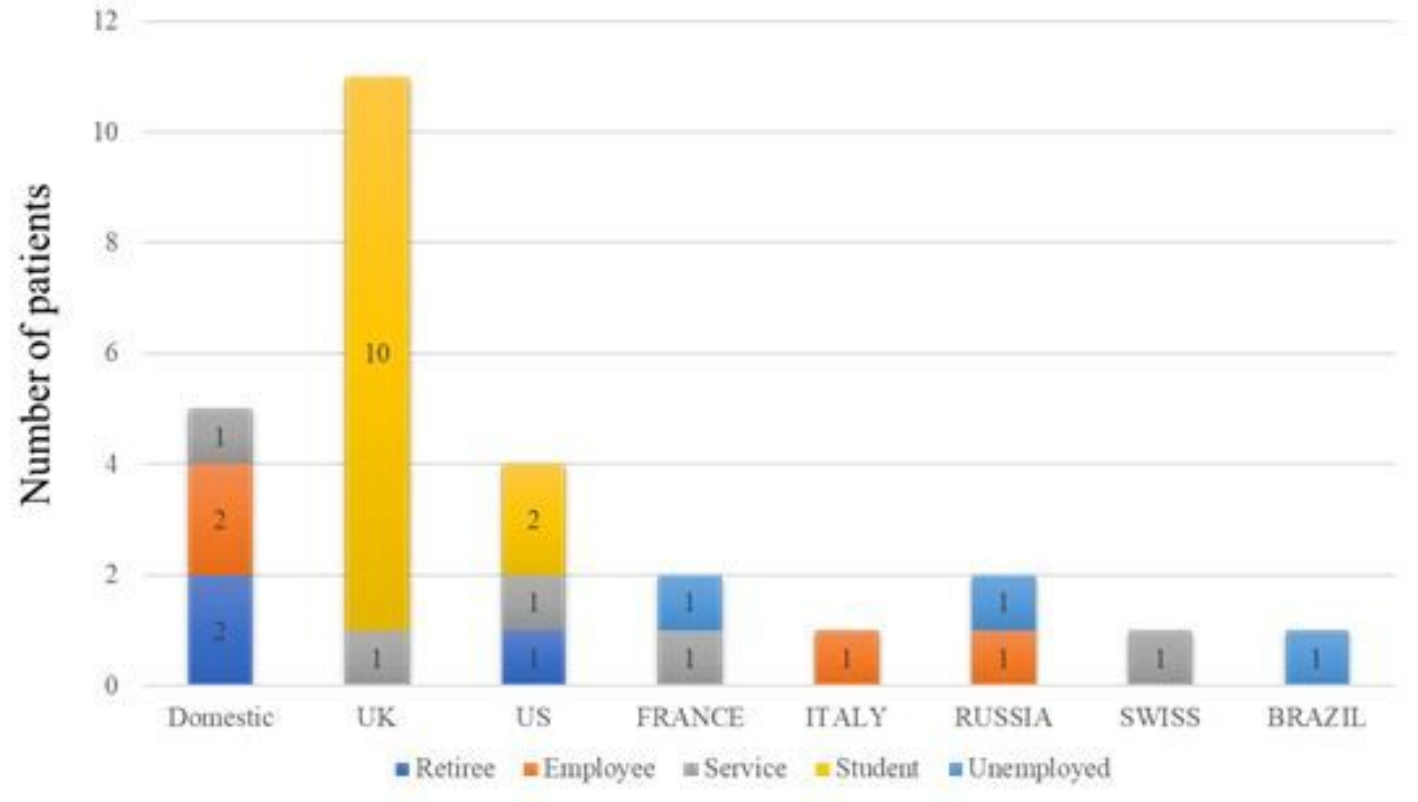

Figure 3

Occupations of abroad cases according to region. The majority of the cases that returned from abroad came from the United Kingdom and were predominantly Chinese students who were studying abroad.

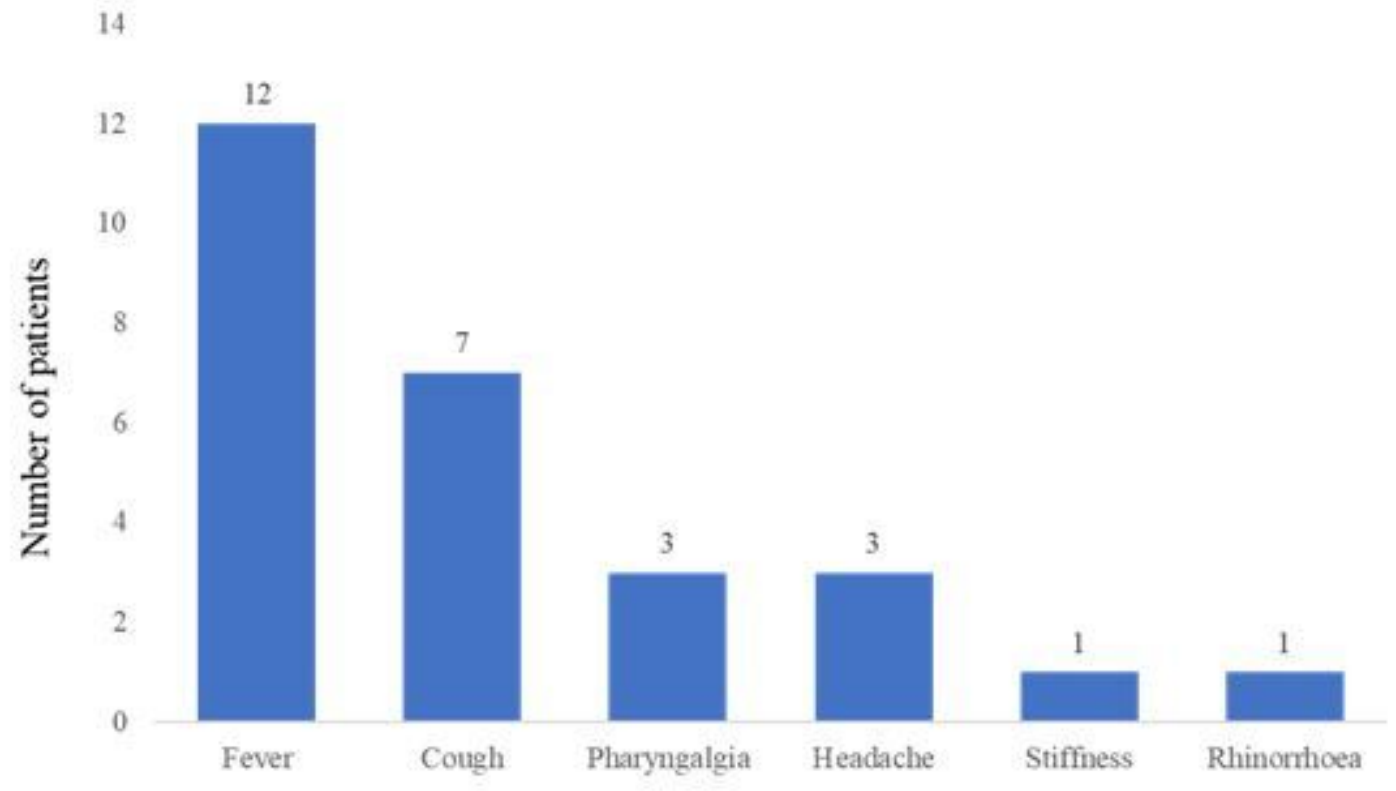

Figure 4 
Symptoms analysis of confirmed cases from the SEH fever outpatient clinic. The main symptom of the 27 confirmed patients was fever, cough, pharyngalgia, headache, muscle ache and rhinorrhea, consistent with the symptoms reported in the extant literature. SEH, Shanghai east hospital

Lymphocyte

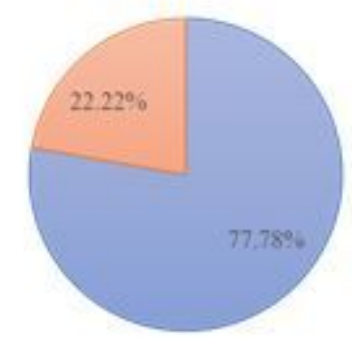

a Normal lymphocyte DLymphopenia
Lung CT

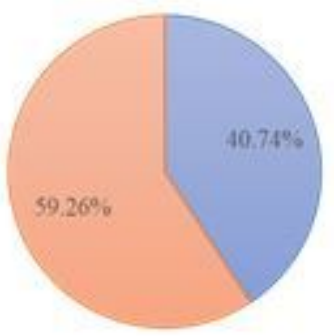

aNormal CT EAbnormal CT

\section{Figure 5}

Laboratory tests and lung CT scan analysis of 27 positive patients. Among 27 confirmed COVID-19 cases, lymphopenia was observed in six patients (22.22\%), and $16(59.26 \%)$ patients had abnormal lung CT scans. CT, computed tomography

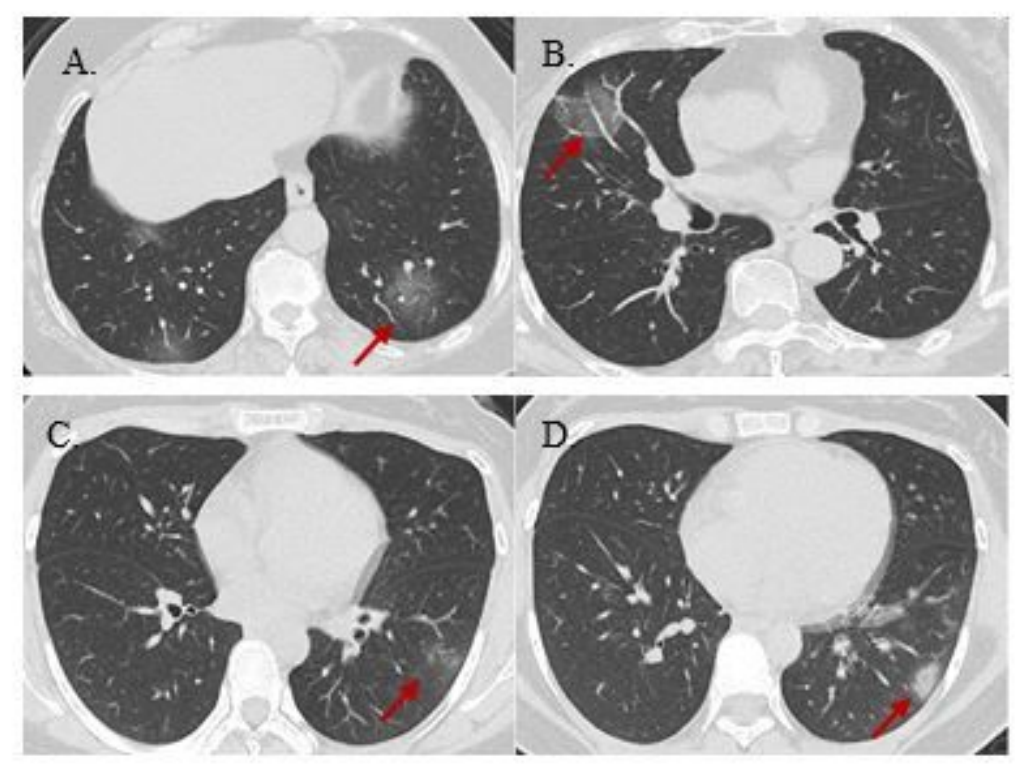

\section{Figure 6}

Typical lung CT findings of confirmed cases. Bilateral ground-glass opacity was marked in the scan A. ground-glass opacity in left lower lobe and multiple mottling; B. typical ground-glass opacity in right middle lobe; C. ground-glass opacity in left lobe; D. patchy shadow in left lobe that indicated inflammatory lesions. (Arrows) CT, computed tomography 